Meta

Journal des traducteurs

Translators' Journal

\title{
Le conteneur
}

\section{Jean Delisle}

Volume 17, numéro 2, juin 1972

URI : https://id.erudit.org/iderudit/002512ar

DOI : https://doi.org/10.7202/002512ar

Aller au sommaire du numéro

Éditeur(s)

Les Presses de l'Université de Montréal

ISSN

0026-0452 (imprimé)

1492-1421 (numérique)

Découvrir la revue

Citer cet article

Delisle, J. (1972). Le conteneur. Meta, 17(2), 113-117.

https://doi.org/10.7202/002512ar

Ce document est protégé par la loi sur le droit d'auteur. L’utilisation des services d'Érudit (y compris la reproduction) est assujettie à sa politique d'utilisation que vous pouvez consulter en ligne.

https://apropos.erudit.org/fr/usagers/politique-dutilisation/
Cet article est diffusé et préservé par Érudit.

Érudit est un consortium interuniversitaire sans but lucratif composé de l’Université de Montréal, l'Université Laval et l'Université du Québec à Montréal. Il a pour mission la promotion et la valorisation de la recherche. https://www.erudit.org/fr/ 


\section{IE CONTENEUR}

C'est au transporteur routier (road haulier) MacLean, de l'Alabama, que l'on doit la mise en ouvre de la conteneurisation (containerization) sur une vaste échelle vers la fin des années 50 . Le conteneur ${ }^{1}$ destiné au trafic international et pouvant emprunter successivement différents modes de transport fut désigné sous le nom de transcontainer. Il semble cependant que le terme n'ait pas été retenu. Par définition, la conteneurisation implique l'interchangeabilité du matériel entre divers modes de transport. C'est pourquoi la distinction que l'on voudrait établir entre container (domaine ferroviaire) et «conteneur» (tous les autres cas) est purement arbitraire et sans fondement.

\section{ANATOMIE DU CONTENEUR}

Le conteneur trouve l'un de ses plus proches ancêtres dans le cadre (crate). Dans le jargon des quais, on l'a baptisé box ou tin box. Le conteneur se compose d'une ossature, généralement en acier, constituée par les longerons supérieurs ou longerons du toit (top rail, roof rail), les longerons inférieurs (bottom rail), les longerons avant (front rail), les longerons arrière (rear rail) et les montants d'angles (corner structure ou corner post), ces derniers reliant les structures du toit et de la base. Le cadre latéral (side frame) se compose donc de chacun des

1. Les rédacteurs d'articles de revues (ex. : les Industries françaises d'équipement, Usine nouvelle, Sciences et vie) emploient la graphie anglaise container, tandis que les organismes officiels de normalisation (I.S.O., A.F.N.O.R., C.E.T.T.F.) proposent de franciser l'orthographe en "conteneur *. Soulignons d'autre part que tous les principaux dictionnaires et les grandes encyclopédies ne donnent que la forme container, à l'exception du Dictionnaire encyclopédique Quillet qui relève les deux formes. L'usage, seul juge en ce domaine, se chargera de fixer l'orthographe de ce mot. 
éléments du conteneur parallèles à son axe longitudinal et est constitué des montants d'angles et des longerons supérieurs et inférieurs. Le cadre d'extrémité (end frame) est formé de chacun des éléments du conteneur perpendiculaires à son axe longitudinal et constitué des membrures d'angles et des membrures d'extrémités de la base et du toit. Les côtés de l'enceinte du conteneur forment les parois latérales (side wall) par opposition aux parois d'extrémités (end wall). Ces parois peuvent avoir une porte latérale (side door), ou une porte d'extrémité (end door).

Le plancher repose sur des traverses ou membrures traversières (floor cross member ou simplement cross). De gros anneaux (bull ring) sont fixés dans le plancher pour le saisissage ou arrimage (securing) des machines. Sous les planchers, on trouve parfois des patins (skids) pour faciliter la manutention.

Voilà quels sont les principaux éléments constitutifs du conteneur. Un certain nombre de pièces ou d'évidements viennent s'ajouter à cette boîte métallique pour faciliter la manutention : l'empotage (stuffing) ou le dépotage (stripping).

Les portes sont munies d'une crémone (door lock bar), sorte d'espagnolette composée d'une tige de fer qu'on hausse ou qu'on baisse en faisant tourner une poignée.

Sur les parois intérieures sont disposées des boucles (rope ring) dans lesquelles passent les cordages ou sangles qui immobiliseront la marchandise arrimée à l'intérieur. Aux angles du conteneur, on trouve les pièces de coin (corner fitting ou corner casting) qui assurent la liaison entre les montants et les longerons. On les appelle aussi coins de toiture ou coins de plancher ${ }^{2}$, selon le cas. On utilise les quatre coins des angles supérieurs dans les opérations de levage. Le palonnier (spreader) des engins de levage est au gabarit de la toiture du conteneur ; il comporte quatre verrous télécommandés qui s'engagent dans les orifices des coins et permet ainsi d'exercer les efforts de hissage dans le prolongement des montants, ce qui évite toute déformation du conteneur. Des oilletons de levage (lifting eye) ou d'arrimage (securing eye) sont fixés au conteneur et se composent essentiellement d'un anneau ou d'une bride. À la partie supérieure arrière d'un conteneur à toit ouvrant, il y a un longeron transversal mobile qui forme la têtière (header). Enfin, dans le plancher de certains conteneurs sont aménagés des tunnels transversaux (fork lift pockets ou simplement pockets) que l'on désigne aussi par entrées de fourches. Ces évidements permettent le passage des deux branches de la fourche d'un chariot frontal.

\section{TYPES DE CONTENEURS}

Les transporteurs mettent à la disposition de leurs clients différents types de conteneurs pour répondre à leurs besoins les plus variés. Le conteneur ordinaire (straight box) ne comporte pas d'accessoires sous le plancher comme le conteneur flexi-van (flexi-van container). À ce dernier, il ne manque qu'un

2. Source : Compagnie générale transatlantique. 
essieu pour être transformé en remorque routière. Le dessous du plancher porte les pièces suivantes : la jupe (skirt), les béquilles (landing gear supports, leg ou supporting stilts), la cheville d'attelage à l'avant (king pin), destinée à s'engager dans la sellette du tracteur ( $f$ ifth wheel), les câbles pour le freinage des essieux et les câbles et feux de signalisation. Il faut aussi mentionner le talon (foot pad spacer) qui se fixe sous le coin-plancher pour surélever le conteneur afin que les accessoires ne portent pas au sol ou sur la toiture d'un autre conteneur.

Un conteneur à toit ouvrant (open top container) ou conteneur bâché (tarpaulin-covered container) se dit d'un conteneur dont la toiture est remplacée par une bâche (tarpaulin ou tarp) fixée sur des arceaux mobiles (roof bows). Le flat (flat) est un conteneur sans parois latérales ni toiture dont les hayons (tail gate) avant et arrière sont rabattables, permettant ainsi d'empiler cinq flats vides dans le volume d'un seul. De même le conteneur repliable ou démontable (collapsible ou folding freight container) offre l'avantage d'être aisément replié ou démonté, puis réassemblé pour une nouvelle utilisation, contrairement au conteneur non repliable (non-collapsible container) dont les éléments sont assemblés à demeure. Relevons également le conteneur-citerne (tank container), le conteneur sec (dry van), le conteneur réfrigéré (reefer van), le conteneur de mi-hauteur (half-container), c'est-à-dire haut de quatre pieds au lieu de huit, mais de longueur et largeur standards. On désigne parfois les conteneurs de vingt pieds et ceux de quarante pieds par les expressions twenty footer et forty footer. Enfin, les dravos ou odd container sont des conteneurs de diverses tailles, non normalisés.

Disons quelques mots, pour finir, des techniques de manutention des conteneurs. La manutention verticale (lift-on/lift-off ou lolo) désigne la manutention d'unités de charge par déplacement vertical, donc à l'aide d'un portique (travellinggantry crane), d'une grue à flèche (gantry crane) ou d'un ponton flottant. La manutention horizontale (roll-on/roll-off ${ }^{3}$ ou roro) s'exécute par déplacement horizontal à l'aide d'un tracteur-pousseur (tugmaster). Le fret roro se divise en deux grandes catégories : a) le roro direct, quand il peut se mouvoir lui-même ou être remorqué $; b$ ) le roro indirect, quand il faut le charger sur des remorques esclaves. Dans un précédent numéro de META ${ }^{4}$, on proposait de traduire roll-on/ roll-off par roulage direct ou encore de créer le néologisme «transroulage». Les revues spécialisées (mentionnées à la note 1) n'emploient guère que l'expression manutention horizontale, sans doute parce qu'elle permet de mieux distinguer les deux types de manutention : verticale et horizontale.

Dans le glossaire qui suit, figurent, en plus des mots étudiés, des termes se rapportant au transport des conteneurs, aux terminaux des lignes conteneurisées (containerized routes), aux châssis qui reçoivent les conteneurs ainsi qu'aux charges et efforts appliqués sur les diverses parois des conteneurs.

3. La technique du roll-on/roll-off est née lorsque les Anglais décidèrent, pour leurs livraisons outre-Manche, de charger les camions de transport sur des car-ferries à l'instar des voitures de tourisme.

4. XIV, 3 (1969), p. 161. 
barge-carrying vessel

box

bull ring

certificate of shippability

chassis

collapsible freight container

containerization

container ship

containerized freight

containerized route

corner casting

corner fitting

corner post, corner structures

crate

cross member

door lock bar

door to door

door to pier

dravos

dry van

end door

end frame

end load

end wall

fifth wheel

flat

flexi-van

floor cross member

floor load

folding lock

foot pad (spacer)

(fork lift) pocket

fork pocket

forty footer

gantry crane

gooseneck chassis

half-container

harbour terminal

header

house to house

house to pier

international container traffic

king pin

landing gear supports

leg

lifting eye

lift-on/lift-off, lolo

non-collapsible freight container

odd container

open top containcr

overhead gantry

overhead crane

pier to house navire porte-barges

navire-kangourou

conteneur

gros anneau

certificat de navigabilité

châssis

conteneur repliable ou démontable

conteneurisation

navire porte-conteneur

fret conteneurisé

ligne conteneurisée

coin de toiture, de plancher

pièce de coin, coin de toiture, de plancher

montant (d'angles)

cadre (de déménagement)

voir floor cross member

crémone

porte à porte

porte à quai

conteneur non normalisé

conteneur sec

porte d'extrémité

cadre d'extrémité

charges (sur les parois) d'extrémité

paroi d'extrémité

sellette

flat

conteneur flexi-van

traverse du plancher, membrure traversière

efforts de plancher

verrou rabattable

talon

tunnel transversal, entrée de fourches

voir fork lift pocket

conteneur de quarante pieds

grue à flèche (sur rail)

châssis en col de cygne

conteneur de mi-hauteur

terminal portuaire

têtière

domicile-domicile

domicile à quai

acheminement des conteneurs

en trafic international

cheville d'attelage

béquilles

béquilles

ailleton de levage

manutention verticale

conteneur non repliable

conteneur non normalisé

conteneur à toit ouvrant

portique

portique

quai à domicile 


\begin{tabular}{l} 
pier to pier \\
rail \\
rail terminal \\
reefer van \\
road haulier \\
road terminal \\
roll-on/roll-off, roro \\
roof bow \\
roof load \\
roof rail \\
rope ring \\
securing eye \\
semi-container vessel \\
shipper \\
side door \\
side frame \\
side load \\
side wall \\
skid \\
skirt \\
spacer \\
spreader \\
stacking \\
straddle carrier \\
straight box \\
stripping \\
stuffing \\
superimposed load \\
supporting stilts \\
tank container \\
tarp/tarpaulin \\
tarpaulin-covered container \\
telescopic spreader bar \\
tin box \\
transhipment \\
transport line \\
travelling-gantry crane \\
tugmaster \\
twenty footer \\
twist lock \\
\\
\hline
\end{tabular}

quai à quai

longeron

gare centre, terminus

conteneur réfrigéré

transporteur routier

gare routière

manutention horizontale

arceau

charges sur le toit

longeron du toit

boucle

œilleton d'arrimage

bâtiment semi-porte-conteneurs

chargeur

porte latérale

cadre latéral

charges (sur les parois) latérales

paroi latérale

patin

jupe

entretoise

palonnier

gerbage, empilage

chariot cavalier, à califourchon

conteneur ordinaire

dépotage

empotage

charges de gerbage

béquilles (de soutien)

conteneur-citerne

bâche

conteneur bâché

palonnier télescopique

conteneur

rupture de charge

chaîne des transports

portique

tracteur-pousseur

conteneur de vingt pieds

verrou rotatif 